\title{
BMJ Open Dairy product consumption and development of cancer: an overview of reviews
}

\author{
Maya M Jeyaraman, ${ }^{1,2}$ Ahmed M Abou-Setta, ${ }^{1,2}$ Laurel Grant, ${ }^{3}$ Farnaz Farshidfar, ${ }^{1}$ \\ Leslie Copstein, ${ }^{1}$ Justin Lys, ${ }^{1}$ Tania Gottschalk, ${ }^{4}$ Danielle Desautels, ${ }^{3,5,6}$ \\ Piotr Czaykowski, ${ }^{2,3,5,6}$ Marshall Pitz, ${ }^{2,3,5,6,7}$ Ryan Zarychanski ${ }^{1,2,3,5,6,7}$
}

To cite: Jeyaraman MM, Abou-Setta AM, Grant L, et al. Dairy product consumption and development of cancer: an overview of reviews. BMJ Open 2019;9:e023625. doi:10.1136/ bmjopen-2018-023625

- Prepublication history and additional material for this paper are available online. To view these files, please visit the journal online (http://dx.doi. org/10.1136/bmjopen-2018023625).

Received 17 April 2018 Revised 21 November 2018 Accepted 28 November 2018

Check for updates

(c) Author(s) (or their employer(s)) 2019. Re-use permitted under CC BY-NC. No commercial re-use. See rights and permissions. Published by BMJ.

For numbered affiliations see end of article.

Correspondence to Dr Maya M Jeyaraman; maya.jeyaraman@umanitoba.ca

\section{ABSTRACT}

Objectives To provide a comprehensive systematic overview of current evidence from pooled analyses/meta-analyses and systematic reviews (PMASRs) pertaining to dairy consumption and incident cancer and/or all-cause or cancer-specific mortality.

Design Overview of reviews.

Setting Community setting.

Participants The unit of analysis is PMASRs. A total of 42

PMASRs was included in this overview of reviews.

Interventions/exposures Any dairy product consumption (eg, milk, yogurt, etc).

Primary and secondary outcomes measures Primary outcome measure is development of any type of cancer. Secondary outcome measures are all-cause mortality and cancer-specific mortality.

Results From 9693 citations identified, we included 42 PMASRs (52 study reports) published between 1991 and 2017. Thirty-one (74\%) of these was pooled analyses/ metaanalyses, and only $11(26 \%)$ were systematic reviews and meta-analyses. There was a wide variability in the type of study designs included within the other PMASRs, thus contributing to variable and, in instances, divergent estimates of cancer risk for several cancer subtypes. For example, only one systematic review and meta-analysis exclusively included prospective study designs. Most PMASRs were of low to moderate quality based on the Assessing the Methodological Quality of Systematic Reviews (AMSTAR) scores. The median AMSTAR score was 5 (IQR 2-7). Our overview identified conflicting evidence from PMASRs on association between dairy consumption and incident cancers or mortality. Heterogeneity in summary estimates reflected the inclusion of variable study designs and overall low methodological quality of individual PMASRs.

Conclusions The association between dairy consumption and cancer risk has been explored in PMASRs with a variety of study designs and of low to moderate quality. To fully characterise valid associations between dairy consumption and risk of cancer and/or mortality rigorously conducted, PMASRs including only high-quality prospective study designs are required.

Trial registration number CRD42017078463.

\section{INTRODUCTION}

Cancer is a major public health issue in North America and worldwide, ${ }^{1}$ with a global burden

\section{Strengths and limitations of this study}

To the best of our knowledge, this is the first overview of reviews to synthesise current evidence from pooled analyses/meta-analyses and systematic reviews (PMASRs) that evaluated associations between dairy consumption and development of incident cancer and/or mortality.

- Comprehensive search strategy and a systematic review methodology.

- An a priori protocol registered in Prospective Register of Systematic Reviews.

- Only included English-language PMASRs.

- Marked heterogeneity among included PMASRs with respect to study design and methodological quality of included studies limits ability to reach valid conclusions regarding associations or causal inferences.

that is expected to grow to 21.7 million new cases and 13 million cancer deaths by $2030 .^{2}{ }^{3}$ Cancer is the leading cause of mortality in Canada $(30.2 \%)^{4}$ and the second leading cause of mortality in the USA. ${ }^{1}$ Many risk factors such as genetic inheritance, diet, physical inactivity, smoking, alcohol consumption and exposure to environmental pollution and radiation have been associated with the development of cancer. ${ }^{5}$ Only 5\%-10\% of cancers are attributed to inherited genetic defects, ${ }^{5}$ while the remaining $90 \%-95 \%$ of all cancers are attributed to either environmental or lifestyle factors. ${ }^{5}$ A direct relationship between diet and an increased risk of cancer has been well described. It is estimated that diet accounts for $35 \%$ of all cancer risk. ${ }^{6}$

Milk and dairy products from various animal sources are considered well-balanced nutritive foods, ${ }^{7}$ and dietary guidelines recommend a daily consumption of low-fat dairy products for optimal health. ${ }^{8}$ Recently, however, the assumed beneficial effects of dairy consumption have been questioned and potential risks have been identified by nutrition scientists ${ }^{9}$ 
and authors. ${ }^{10} 11$ While dairy products are believed to be rich in bioactive compounds that may be beneficial to health, ${ }^{72}$ studies have also suggested that dairy may contain harmful reproductive and cancer-causing hormones ${ }^{13} 14$ and that regular dairy consumption may promote cancer by increasing insulin-like growth factor levels. ${ }^{15}$ Several epidemiological studies have explored the association between dairy consumption and risk of various cancers but the evidence from primary research remains inconclusive and controversial. ${ }^{716-18}$

Numerous pooled analyses/meta-analyses and systematic reviews (PMASRs) have synthesised a variety of data-exploring associations between dairy products consumption and cancer incidence. ${ }^{19-23}$ The scope, objectives, outcomes and the methodological quality of these PMASRs are diverse. In order to inform clinical decision-making and to guide future research, we conducted an overview of reviews to identify the totality of evidence currently available from PMASRs. Our objective is to provide a systematic and comprehensive critical appraisal of synthesised evidence evaluating the association of dairy consumption and incident cancer and all-cause or cancer-specific mortality.

\section{METHODS}

We conducted this overview of reviews using an a priori protocol (CRD42017078463) ${ }^{24}$ (online supplementary file) and reported it according to the Preferred Reporting Items for Systematic Reviews and Meta-Analyses (PRISMA) ${ }^{25}$ The eligibility criteria are reported in online supplementary table 1.

\section{Search methods}

We searched for PMASRs on dairy consumption and cancer using a peer-reviewed comprehensive search strategy of the following bibliographic databases: PubMed (National Library of Medicine), Medline (Ovid), EMBASE (Ovid), Cochrane Library (Wiley), Centre for Agriculture and Biosciences (CAB) abstracts (Ovid), Food, Science $\&$ Technology (Ovid) and Cumulative Index to Nursing and Allied Health Literature (CIHAHL) (EbscoHost) from inception to July 2017, irrespective of the publication status or publication year. We also searched Web of Science-Science Citation Index (Clarivate Analytics), Web of Science-Conference Abstracts (Clarivate Analytics) and Scopus (Elsevier). To identify additional potentially relevant studies, we performed forward searches in Scopus (Elsevier) and Web of Science (Clarivate Analytics). We hand-searched conference proceedings (2014-2017) for the following conferences: American Society of Clinical Oncology's annual meeting (ASCO), American Association for Cancer Research (AACR) Annual Meeting, and European Society for Medical Oncology (ESMO). We contacted experts in the field and searched Prospective Register of Systematic Reviews (PROSPERO) for relevant unpublished reviews. We used EndNote (V.X7, Thomson Reuters) for reference management. Our search strategy for Medline is reported in online supplementary table 2. We used the study design filter for systematic reviews and meta-analysis in our search strategies formatted for the various databases.

\section{Study selection process}

Two reviewers (MJ and one of LG, JL or AS) independently screened the titles and abstracts of citations identified by our search strategy. Each screened abstract was marked as either, included, excluded or unsure. Conflicts were resolved using consensus or third-party adjudication. Full texts were retrieved for the citations marked as either included or unsure. Full-text screening was performed by two reviewers (LG and either FF or LC) and conflicts were again resolved either through consensus or third-party adjudication. A PRISMA flow diagram illustrating the number of studies either included or excluded at each step of the study selection process is reported in figure 1 .

\section{Dealing with companion publications}

In the event of companion reports of an included PMASR, we used the publication that contained the most complete information relevant to our overview of reviews as the primary report and listed all other companion publications as secondary reports under the primary reference of the included PMASR.

\section{Assessment of methodological quality of included reviews}

We used the Assessing the Methodological Quality of Systematic Reviews (AMSTAR) ${ }^{26}$ tool to assess the methodological quality/internal validity of each of the included PMASRs. Two reviewers (LG and either of FF or LC) independently assessed the quality of the included PMASRs and resolved conflicts either through consensus or third party adjudication (figure 2).

\section{Data extraction and analysis}

We developed, piloted and used customised data extraction forms to extract data from included PMASRs. Two review authors (LG and either of FF or LC) independently extracted the characteristics and the summary effect estimates and CIs with conflicts resolved through consensus or third-party adjudication. The individual study characteristics extracted from the included PMASRs are itemised in table 1 and online supplementary table 3 . We analysed the extracted study characteristics using Microsoft Excel (Excel V. 14, Microsoft, Redmond, Washington, USA). Categorical and continuous data from included PMASRs are reported as proportions or medians/means with accompanying measures of variance where appropriate. The summary effect estimates from 156 meta-analyses (comparing highest vs lowest dairy intake) reported by the included PMASRs were extracted and are reported in online supplementary tables 4,5 and 6 . We assessed the nature of association for each of the meta-analysis reported in the PMASRs based on the extracted summary effect estimates and the CIs.

The number of studies reporting the nature of association between dairy consumption and each type of cancer 


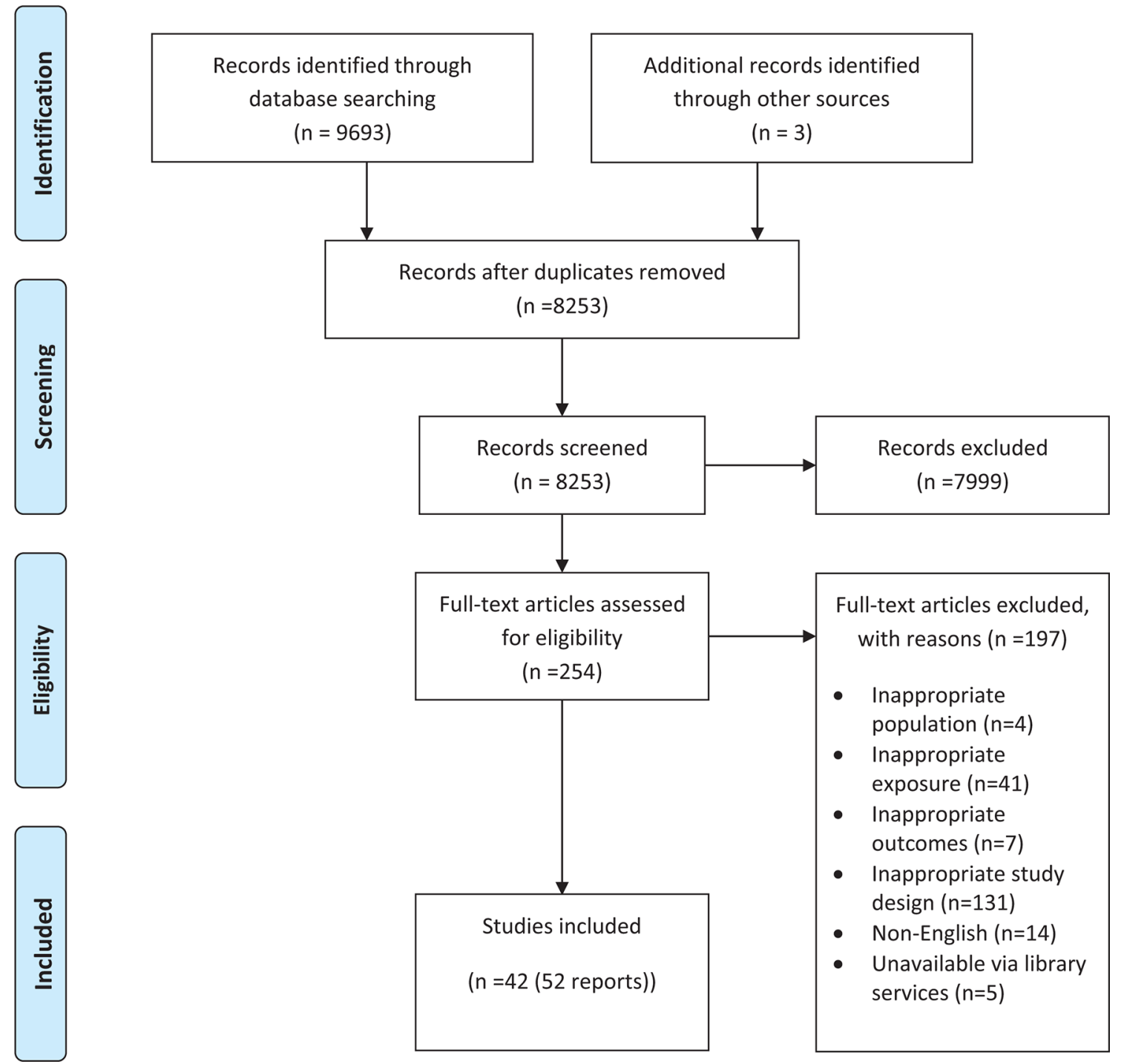

Figure 1 Preferred Reporting Items for Systematic Reviews and Meta-Analyses flow diagram depicting study selection process.

outcome has been reported using radar plot in figure 3 . The number of studies reporting the nature of association between various dairy products and specific gastrointestinal or hormone dependent cancer is reported using radar plot in figures 4 and 5 .

\section{Patient involvement}

Patients were not involved in this overview of reviews.

\section{RESULTS}

\section{Search results}

We identified 9693 unique records from searching electronic databases and 3 additional records through handsearching; 42 PMASRs (52 reports) met our inclusion criteria and were included in this overview of reviews (figure 1).

\section{Description of included PMASRs}

The included PMASRs were published between 1991 and 2017. In total, 31 (74\%) were pooled analyses/ meta-analyses and $11(26 \%)$ were systematic reviews and meta-analyses. The $11^{19} 20^{27-35}$ systematic reviews and meta-analysis reported on the risks of colorectal cancer, ${ }^{27} 3133$ prostate cancer, ${ }^{20}$ lung cancer, ${ }^{19}$ breast cancer, ${ }^{2835}$ gastric cancer, ${ }^{32} 34$ ovarian cancer ${ }^{29}$ or oesophageal cancer. ${ }^{30}$ One systematic review ${ }^{33}$ was included as three separate PMASRs because the data were reported separately for three different patient populations based on their ability to digest lactose. The PMASRs included many study designs such as cohort, case-control, nested-case-control and case-cohorts (online supplementary table 3$)$. Only 1 (2\%) of the 11 systematic reviews and meta-analysis exclusively included prospective cohort studies. The PMASRs included primary studies that were conducted in various continents (online supplementary table 3). The number (\%) of PMASRs that included primary studies from different continents is as follows: Europe $(\mathrm{n}=39(93 \%))$, North America $(\mathrm{n}=35(83 \%))$, Asia $(n=27(64 \%))$, South America $(n=13(31 \%))$ or Oceania $(\mathrm{n}=9(21 \%))$. Most PMASRs $(\mathrm{n}=26(62 \%))$ were 


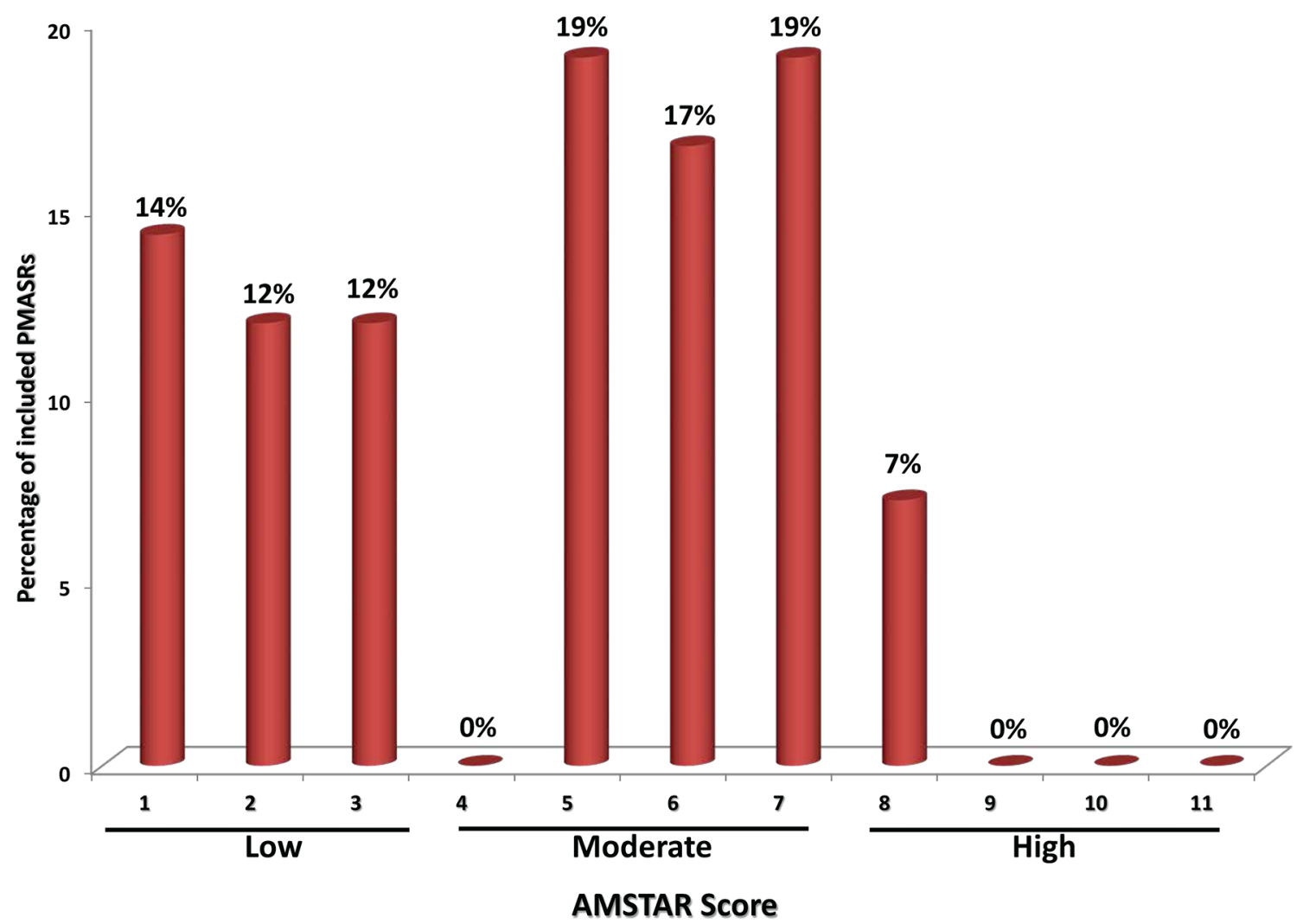

Figure 2 Bar chart depicting the Assessing the Methodological Quality of Systematic Reviews (AMSTAR) scores of included pooled analyses/meta-analyses and systematic reviews (PMASRs). The $y$-axis represents the percentage of included PMASRs, and the x-axis represents the AMSTAR scores (low, moderate or high).

funded by non-industry sources; few $(\mathrm{n}=2(5 \%))$ were industry sponsored and the remaining $(\mathrm{n}=14(33 \%))$ did not report a source of funding. Informative subgroup analyses, evaluation of publication bias and meta-regression based on important potential confounders were performed in $62 \%, 55 \%$ and $33 \%$ of PMASRs, respectively. Most of the PMASRs reported using random-effects model $(60 \%)$ for their analyses; $7 \%$ used fixed-effects models and 19\% reported using both models. The key features and the individual characteristics of the included PMASRs are summarised in table 1 and online supplementary table 3 .

\section{Methodological quality of included reviews}

The AMSTAR scores ranged from 1 to 8 out of a possible maximum score of 11 . The median score and the IQR were 5 and (2.25-6.75), respectively. Thirty-eight per cent $(16 / 42)$ of the PMASRs had a score of 1-3 (low quality), and $55 \%(23 / 42)$ had a score of 4-7 (moderate quality). Only 7\% (3/42) of PMASRs had a high score of 8 (high quality), with none reaching the maximum score of 11 (figure 2 and online supplementary table 7 ). The 11 included systematic reviews and meta-analyses were mostly of moderate quality ${ }^{19} 27-35$ with 2 that were low $^{35}$ and high quality. ${ }^{33}$ The domains of the AMSTAR tool in which most PMASRs scored the lowest were for search of 'grey literature' or 'unpublished literature', providing a list of included and excluded studies, and providing source of funding for each of the included primary studies in the PMASR. The domains of the AMSTAR tool in which most PMASRs scored the highest were for providing characteristics of included studies and using appropriate methods for pooling data from primary studies. The relationship between AMSTAR scores and the publication year of the included PMASRs is reported using a line plot in online supplementary figure 1 .

\section{Primary outcome}

Nature of association between dairy consumption and risk of cancer

The various dairy products $(n=19)$ reported as exposures in the PMASRs were all-dairy products, whole milk, milk, fermented milk, non-fermented milk, low-fat milk, skimmilk, yogurt, cheese, hard cheese, cottage cheese, butter, solid cheese, dairy calcium, ice cream, fermented dairy, low-fat dairy, high-fat dairy and lactose. We grouped the reported cancer outcomes $(n=14)$ in the PMASRs into gastrointestinal cancers (oesophageal, gastric, pancreatic and colorectal cancers); hormone-dependent cancers (prostate, ovarian, endometrial and breast cancers); other cancers (bladder cancer, renal cell cancer, lung cancer, thyroid cancer, non-Hodgkin's lymphoma and multiple myeloma).

Out of 153 reported meta-analyses (comparing highest vs lowest dairy consumption) in the 42 PMASRs, 109 $(71 \%)$ showed no evidence of a statistically significant 
Table 1 Key features of the included pooled analyses/meta-analyses and systematic reviews (PMASRs)

\begin{tabular}{|c|c|c|c|}
\hline \multirow[b]{2}{*}{ Included PMASRs } & \multirow[b]{2}{*}{$\mathbf{N}(\%)$} & \multicolumn{2}{|l|}{ Range } \\
\hline & & Minimum & Maximum \\
\hline Included PMASRs, $\mathrm{n}$ & $42(100)$ & - & - \\
\hline Included meta-analysis, $\mathrm{n}$ & $31(74)$ & - & - \\
\hline Included systematic review and meta-analysis, $\mathrm{n}$ & $11(26)$ & - & - \\
\hline $\begin{array}{l}\text { Systematic review and meta-analysis that only included prospective } \\
\text { cohorts, } n\end{array}$ & $1(2)$ & - & - \\
\hline PMASRs that only included prospective cohorts, $n$ & $7(17)$ & - & - \\
\hline Publication year & - & 1991 & 2017 \\
\hline Funding (industry-funded PMASRs) & $2(5)$ & - & - \\
\hline Funding (non-industry-funded PMASRs) & $26(62)$ & - & - \\
\hline Funding (no funding/not reported) & $14(33)$ & - & - \\
\hline Age (years) & - & 8 & 107 \\
\hline PMASRs that used random-effects model, $n$ & $25(60)$ & - & - \\
\hline PMASRs that used fixed-effects model, $n$ & $3(7)$ & - & - \\
\hline PMASRs that used random-effects and fixed-effects models, $n$ & $8(19)$ & - & - \\
\hline PMASRs that reported subgroup analysis, $n$ & $26(62)$ & - & - \\
\hline PMASRs that reported publication bias, $n$ & $23(55)$ & - & - \\
\hline PMASRs that reported meta-regression, $\mathrm{n}$ & $14(33)$ & - & - \\
\hline $\begin{array}{l}\text { PMASRs that reported Grading of Recommendations Assessment, } \\
\text { Development and Evaluation (GRADE), } n\end{array}$ & $0(0)$ & - & - \\
\hline PMASRs that reported risk of cancer as outcome, $\mathrm{n}$ & $40(95)$ & - & - \\
\hline PMASRs that reported all-cause mortality as outcome, $n$ & $1(2)$ & - & - \\
\hline PMASRs that reported cancer-specific mortality as outcome, $n$ & $3(7)$ & - & - \\
\hline $\begin{array}{l}\text { AMSTAR scores of PMASRs (out of } 11 \text { ) } \\
\text { AMSTAR, Assessing the Methodological Quality of Systematic Revie }\end{array}$ & - & 1 & 8 \\
\hline
\end{tabular}

association between dairy consumption and incidence of cancers, $20(13 \%)$ showed decreased risk of cancers with dairy consumption and $24(16 \%)$ showed increased risk of cancers with dairy consumption. The nature of associations between various dairy product exposures and the risk of cancer is depicted in table 2 and figure 3 .

\section{Nature of association between dairy consumption and gastrointestinal cancers}

The nature of association between various dairy exposures and the risk of gastrointestinal cancers is depicted in figure 4. Out of 14 cancer outcomes reported in the included PMASRs, the following 4 were gastrointestinal cancers.

\section{Oesophageal cancer}

Six meta-analyses ${ }^{30} 36$ explored associations between various dairy products consumption and risk of oesophageal cancer. Four meta-analyse ${ }^{30}$ showed non-significant associations between 'all-dairy products', milk, cheese or butter consumption and risk of oesophageal cancer. Two meta-analyses ${ }^{30}{ }^{36}$ showed decreased risk of oesophageal cancer with higher yogurt or 'all-dairy products' consumption.

\section{Gastric cancer}

A total of 11 meta-analyses ${ }^{32} 343738$ explored associations between dairy consumption and risk of gastric cancer. Ten $^{32} 3437$ showed non-significant associations between 'all-dairy products', milk, yogurt, cheese or butter consumption and risk of gastric cancer. One ${ }^{38}$ meta-analysis showed decreased risk of gastric cancer with higher milk consumption.

Pancreatic cancer

Eight meta-analyses ${ }^{23} 39$ showed non-significant associations between whole milk, milk, low-fat milk, skim milk, yogurt, cheese, cottage cheese or ice cream consumption and risk of pancreatic cancer. None of the dairy products was shown to either decrease or increase the risk of pancreatic cancer.

\section{Colorectal cancer}

Twenty-nine meta-analyses ${ }^{27} 31 \quad 33 \quad 40-43$ explored associations between various dairy products and risk of colorectal cancer. Twenty meta-analyses ${ }^{27313340-43}$ showed non-significant associations between 'all-dairy products', milk, fermented milk, yogurt, cheese, cottage cheese, butter, solid cheese, fermented dairy, low-fat dairy or 


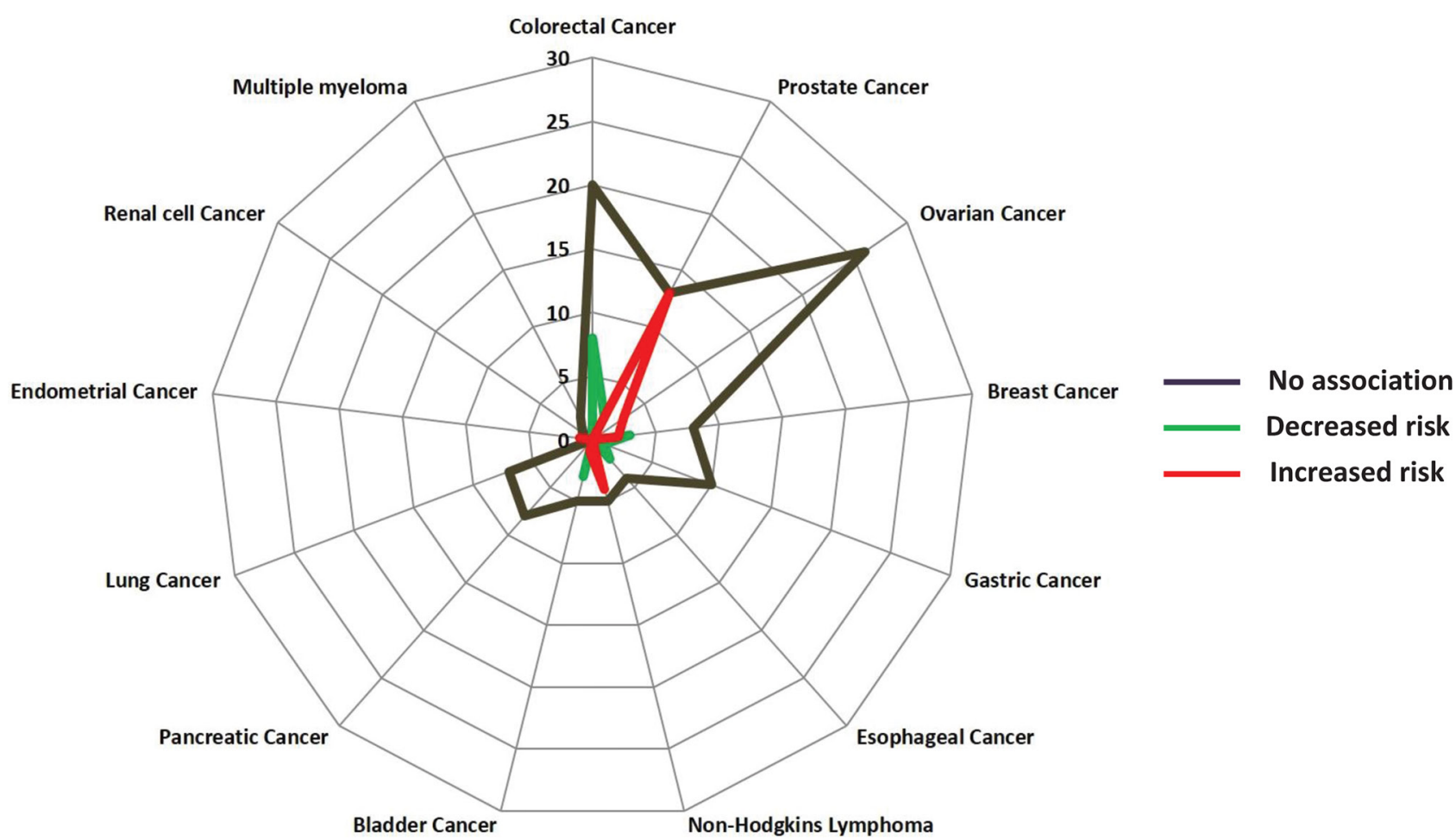

Figure 3 Radar plot depicting the nature of association between dairy consumption and risk of cancer (as reported in included pooled analyses / meta- analyses and systematic reviews (PMASRs)). The numbers on the plot represent the total number of meta-analyses from included PMASRs reporting a specific cancer association. The black, red, and green lines represent number of meta-analyses reporting no association with, increased risk or decreased risk of specific type of cancer, respectively.

high-fat dairy consumption and risk of colorectal cancer. Nine meta-analyses ${ }^{27} 31334043$ showed decreased risk of colorectal cancer with higher consumption of milk, non-fermented milk or 'all-dairy products'.

\section{Evidence from systematic reviews and meta-analysis}

Evidence from available systematic reviews and meta-analyses on oesophageal, ${ }^{30}$ gastric ${ }^{3234}$ or colorectal cancer ${ }^{273133}$ showed either a non-significant association or decreased risk with higher dairy products intake (table 3 and online supplementary table 8).

\section{Nature of association between dairy consumption and hormone-dependent cancers}

The nature of associations between various dairy exposures and the risk of hormone-dependent cancers is depicted in figure 5. Out of 14 cancer outcomes reported in the included PMASRs, the following 4 were hormone-dependent cancers.

\section{Prostate cancer}

Twenty-eight meta-analyses ${ }^{20}{ }^{44-49}$ explored associations between various dairy products consumption and risk of prostate cancer. Thirteen meta-analyses ${ }^{20} 44-4649$ showed non-significant association between 'all-dairy products', milk, skim milk, yogurt, cheese, butter or ice cream consumption and risk of prostate cancer. Two meta-analyses $^{2044}$ showed decreased risk of prostate cancer with higher whole milk and cheese consumption. Thirteen meta-analyses ${ }^{2044} 454748$ showed increased risk of prostate cancer with higher consumption of 'all-dairy products', milk, low-fat milk, cheese or dairy calcium.

\section{Ovarian cancer}

A total of 29 meta-analyses ${ }^{29}{ }^{50-53}$ explored associations between various dairy products consumption and risk of ovarian cancer. Twenty-six meta-analyses ${ }^{29} 50-53$ showed non-significant associations between 'all-dairy products', whole milk, milk, low-fat milk, skim milk, yogurt, cheese, hard cheese, cottage cheese, butter, ice cream or lactose, consumption and risk of ovarian cancer. Three meta-analyses $^{50} 5253$ showed increased risk of ovarian cancer with higher consumption of whole milk or lactose exposure.

\section{Endometrial cancer}

Only one meta-analysis ${ }^{54}$ explored the association between 'all-dairy products' consumption and risk of endometrial cancer. 'All-dairy products' consumption was shown to increase the risk of endometrial cancer in this meta-analysis.

\section{Breast cancer}

Thirteen meta-analyses ${ }^{28} 355556$ explored associations between various dairy products and risk of breast cancer. Eight meta-analyses ${ }^{28} 3555$ showed non-significant associations between 'all-dairy products', whole milk, milk, low-fat milk, cheese, dairy calcium or high-fat dairy consumption and the risk of breast cancer. Three meta-analyses ${ }^{35}$ showed decreased risk of breast cancer with higher consumption of 'all-dairy products', yogurt 
A. Colorectal cancer

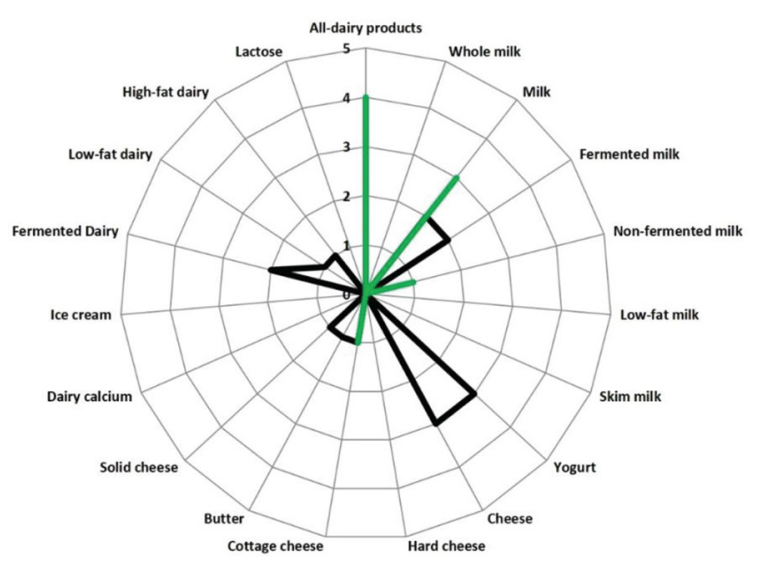

B. Gastric cancer

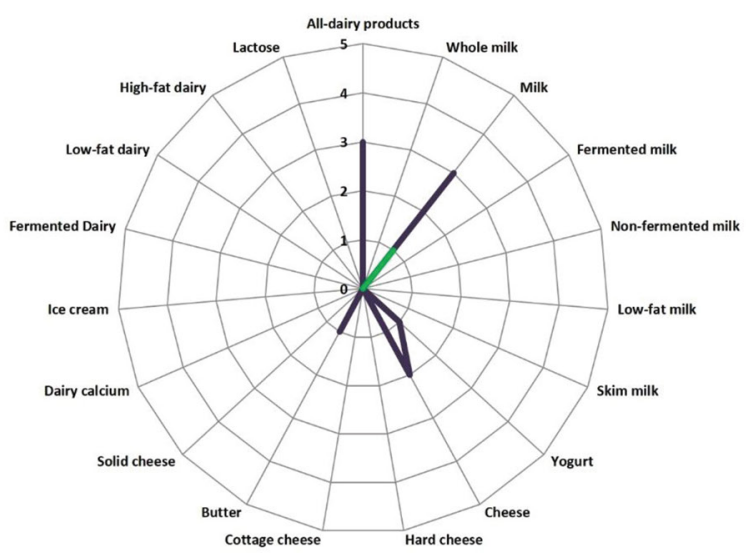

\section{Esophageal cancer}

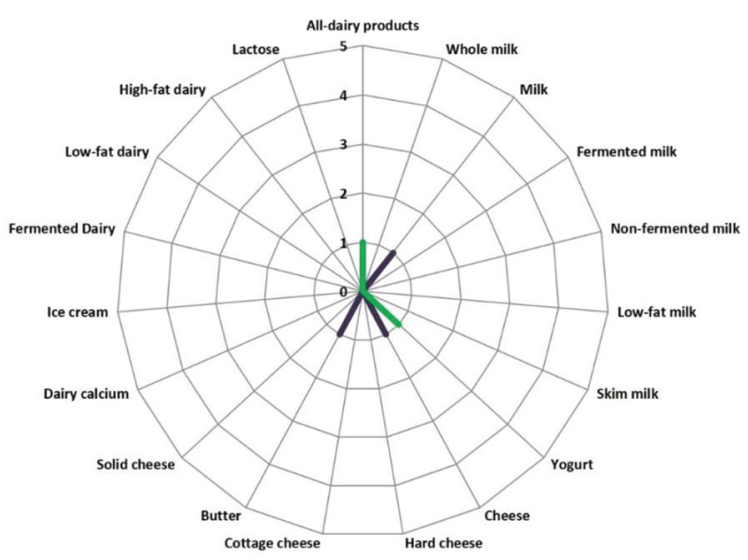

Figure 4 Radar plots depicting the associations between various dairy products consumption and risk of gastrointestinal cancer. The numbers on the circles of each plot represent the total number of meta-analyses from included pooled analyses / meta- analyses and systematic reviews reporting a specific cancer association. The black and green lines represent number of meta-analyses reporting no association with or decreased risk of gastrointestinal cancers, respectively.

or low-fat dairy. Two meta-analyses ${ }^{56}$ showed increased risk of breast cancer with higher consumption of milk or cheese.

\section{Evidence from systematic reviews and meta-analysis}

Evidence from systematic reviews and meta-analysis on prostate cancer, ${ }^{20}$ breast cancer ${ }^{28} 35$ or ovarian cancer ${ }^{29}$ indicates heterogeneous estimates of cancer risk pertaining to dairy consumption (table 3 and online supplementary table 8 ).

\section{Nature of association between dairy consumption and other reported cancers \\ Bladder cancer}

Nine meta-analyses ${ }^{57} 58$ explored associations between various dairy products and risk of bladder cancer. Five meta-analyses ${ }^{57} 58$ showed non-significant associations between 'all-dairy products', milk, cheese or butter consumption and risk of bladder cancer. Three meta-analyses $^{58}$ showed decreased risk of bladder cancer with higher consumption of milk, fermented milk or skim milk. One meta-analysis ${ }^{58}$ showed increased risk of bladder cancer with higher consumption of whole milk.

\section{Renal cell carcinoma}

Only one meta-analysis ${ }^{59}$ explored the association between milk consumption and risk of renal cell carcinoma. Milk consumption was non-significantly associated with risk of renal cell carcinoma in this meta-analysis.

\section{Lung cancer}

Seven meta-analyses ${ }^{19}{ }^{60}$ explored associations between dairy products and risk of lung cancer. All seven meta-analyses showed non-significant associations between 'all-dairy products', milk, low-fat milk, yogurt or cheese consumption and risk of lung cancer.

\section{Non-Hodgkin's lymphoma}

Nine meta-analyses ${ }^{61}{ }^{62}$ explored associations between various dairy products and risk of non-Hodgkin's lymphoma. Five meta-analyses ${ }^{61}{ }^{62}$ showed non-significant associations between 'all-dairy products', milk, 

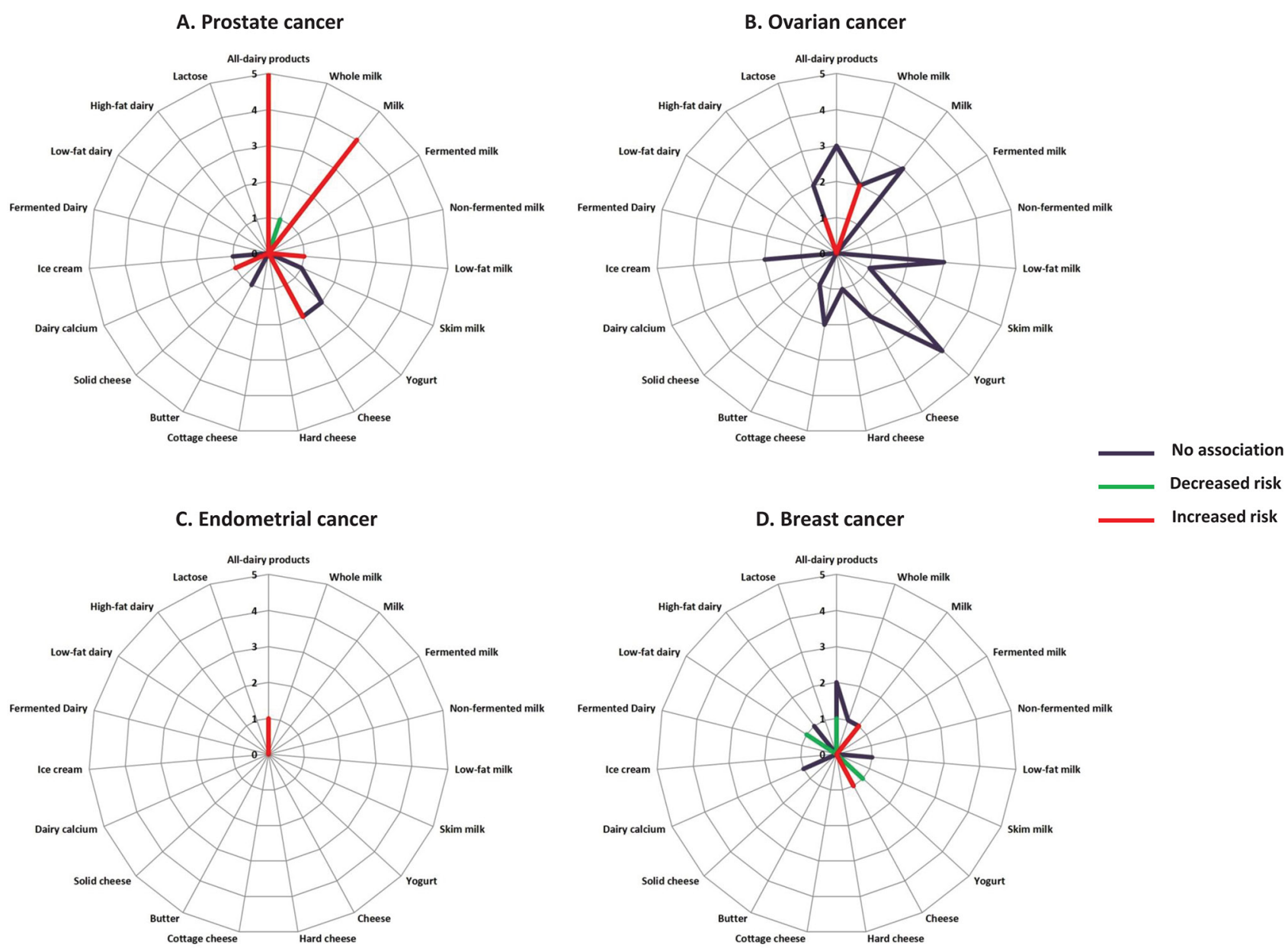

Figure 5 Radar plots depicting the nature of association between various dairy products consumption and risk of hormonedependent cancers. The numbers on the circles of each plot represent the total number of meta-analyses from included pooled analyses/meta- analyses and systematic reviews reporting a specific cancer association. The black, red and green lines represent number of meta-analyses reporting no association with, increased risk or decreased risk of hormone-dependent cancers, respectively.

yogurt or cheese consumption and risk of non-Hodgkin's lymphoma. Four meta-analyses ${ }^{62}$ showed increased risk of non-Hodgkin's lymphoma with consumption of 'all-dairy products', milk, butter or ice cream.

\section{Multiple myeloma}

Two meta-analyses ${ }^{61}$ explored associations between milk or cheese consumption and risk of multiple myeloma and showed non-significant associations.

\section{Thyroid cancer}

Only one PMASR ${ }^{63}$ explored three associations between milk, cheese or butter and risk of thyroid cancer. Because CIs for the summary effect estimates were not reported, the nature of these associations was not assessable.

\section{Evidence from systematic reviews and meta-analysis}

One systematic review on lung cancer ${ }^{19}$ showed non-significant association between dairy consumption and lung cancer risk.

\section{Secondary outcomes}

Nature of association between dairy consumption and the risk of mortality

All-cause mortality

Only one PMASR ${ }^{64}$ reported meta-analyses exploring associations between 'all-dairy products', milk, cheese or butter consumption and risk of all-cause mortality (online supplementary table 8). Non-significant associations were found between 'all-dairy products', milk, cheese or butter consumption and risk of all-cause mortality.

\section{Cancer-specific mortality}

Three PMASRs ${ }^{196465}$ reported non-significant association between milk consumption and cancer-specific mortality (online supplementary table 8). Two PMASRs ${ }^{645}$ reported non-significant association between yogurt, milk, cheese, all-dairy products or butter consumption and risk of cancer-specific mortality. One PMASR ${ }^{19}$ reported a significant decrease in risk of lung cancer-specific mortality with higher consumption of cheese and a non-significant 
Table 2 Nature of association between dairy consumption and risk of cancer reported in included pooled analyses / metaanalyses and systematic reviews

\begin{tabular}{|c|c|c|c|c|c|c|c|c|c|c|c|c|c|c|}
\hline \multirow[b]{2}{*}{ Type of dairy } & \multicolumn{14}{|c|}{ Type of Cancer } \\
\hline & CR & PR & OV & BR & GS & EP & NHL & Lung & EM & BL & PN & TD & $\begin{array}{l}\text { Renal } \\
\text { cell }\end{array}$ & MM \\
\hline \multirow[t]{8}{*}{ All dairy products } & - & + & $\varnothing$ & $\varnothing$ & $\varnothing$ & $\varnothing$ & $\varnothing$ & $\varnothing$ & + & $\varnothing$ & & & & \\
\hline & - & + & $\varnothing$ & - & $\varnothing$ & - & + & $\varnothing$ & & & & & & \\
\hline & $\varnothing$ & + & $\varnothing$ & $\varnothing$ & $\varnothing$ & & & & & & & & & \\
\hline & $-\varnothing$ & & & & & & & & & & & & & \\
\hline & $\varnothing$ & + & & & & & & & & & & & & \\
\hline & - & + & & & & & & & & & & & & \\
\hline & $\varnothing$ & $\varnothing$ & & & & & & & & & & & & \\
\hline & & $\varnothing$ & & & & & & & & & & & & \\
\hline \multirow[t]{4}{*}{ Whole milk } & & - & $\varnothing$ & $\varnothing$ & & & & & & + & $\varnothing$ & & & \\
\hline & & & + & & & & & & & & & & & \\
\hline & & & $\varnothing$ & & & & & & & & & & & \\
\hline & & & + & & & & & & & & & & & \\
\hline \multirow[t]{7}{*}{ Milk } & - & + & $\varnothing$ & + & - & $\varnothing$ & + & $\varnothing$ & & $\varnothing$ & $\varnothing$ & & $\varnothing$ & $\varnothing$ \\
\hline & - & $\varnothing$ & $\varnothing$ & $\varnothing$ & $\varnothing$ & & $\varnothing$ & $\varnothing$ & & - & & & & \\
\hline & - & + & $\varnothing$ & & $\varnothing$ & & & & & & & & & \\
\hline & $\varnothing$ & $\varnothing$ & & & $\varnothing$ & & & & & & & & & \\
\hline & $\varnothing$ & + & & & & & & & & & & & & \\
\hline & & $\varnothing$ & & & & & & & & & & & & \\
\hline & & + & & & & & & & & & & & & \\
\hline \multirow[t]{2}{*}{ Fermented milk } & $\varnothing$ & & & & & & & & & - & & & & \\
\hline & $\varnothing$ & & & & & & & & & & & & & \\
\hline Non-fermented milk & - & & & & & & & & & & & & & \\
\hline \multirow[t]{3}{*}{ Low-fat milk } & & + & $\varnothing$ & $\varnothing$ & & & & $\varnothing$ & & & $\varnothing$ & & & \\
\hline & & & $\varnothing$ & & & & & & & & & & & \\
\hline & & & $\varnothing$ & & & & & & & & & & & \\
\hline Skim milk & & $\varnothing$ & $\varnothing$ & & & & & & & - & $\varnothing$ & & & \\
\hline \multirow[t]{4}{*}{ Yogurt } & $\varnothing$ & $\varnothing$ & $\varnothing$ & - & $\varnothing$ & - & $\varnothing$ & $\varnothing$ & & & $\varnothing$ & & & \\
\hline & $\varnothing$ & $\varnothing$ & $\varnothing$ & & & & & & & & & & & \\
\hline & $\varnothing$ & & $\varnothing$ & & & & & & & & & & & \\
\hline & & & $\varnothing$ & & & & & & & & & & & \\
\hline \multirow[t]{5}{*}{ Cheese } & $\varnothing$ & + & $\varnothing$ & + & $\varnothing$ & $\varnothing$ & $\varnothing$ & $\varnothing$ & & $\varnothing$ & $\varnothing$ & & & $\varnothing$ \\
\hline & $\varnothing$ & $\varnothing$ & $\varnothing$ & $\varnothing$ & $\varnothing$ & & $\varnothing$ & & & $\varnothing$ & & & & \\
\hline & $\varnothing$ & $\varnothing$ & & & & & & & & & & & & \\
\hline & & + & & & & & & & & & & & & \\
\hline & & - & & & & & & & & & & & & \\
\hline Hard cheese & & & $\varnothing$ & & & & & & & & & & & \\
\hline \multirow[t]{2}{*}{ Cottage cheese } & $\varnothing$ & & $\varnothing$ & & & & & & & & $\varnothing$ & & & \\
\hline & - & & $\varnothing$ & & & & & & & & & & & \\
\hline Butter & $\varnothing$ & $\varnothing$ & $\varnothing$ & & $\varnothing$ & $\varnothing$ & + & & & $\varnothing$ & & & & \\
\hline Solid cheese & $\varnothing$ & & & & & & & & & & & & & \\
\hline Dairy calcium & & + & & $\varnothing$ & & & & & & & & & & \\
\hline
\end{tabular}


Table 2 Continued

\section{Type of Cancer}

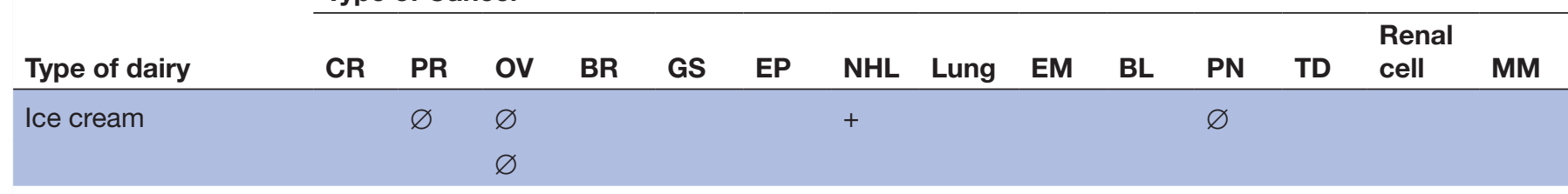

Fermented dairy $\quad \varnothing$

$\varnothing$

\begin{tabular}{lll} 
Low-fat dairy & $\varnothing$ & - \\
High-fat dairy & $\varnothing$ & $\varnothing$ \\
Lactose & $\varnothing$ & \\
& $\varnothing$ & \\
& + \\
\hline
\end{tabular}

$\varnothing$, no association; -, decreased risk of cancer ( $<<0.05) ;+$, increased risk of cancer $(p<0.05)$; BL, bladder; BR, breast; CR, colorectal cancer; EM, endometrial; EP, oesophageal; GS, gastric; MM, multiple myeloma; NHL, non-Hodgkin's lymphoma; OV, ovary; PN, pancreas; PR, prostate; TD, thyroid.

association between milk consumption and lung cancer mortality.

\section{DISCUSSION}

In this overview of reviews, we have provided an up-todate comprehensive critical appraisal of 42 PMASRs (52 reports) that synthesised evidence on dairy consumption and risk of cancer. The current analyses revealed discrepant associations between dairy product consumption and risk of cancer when comparing highest versus the lowest levels of dairy consumption. None of the included primary studies in the PMASRs were randomised controlled trials (RCTs); thus we are only able to infer associations and not causality. In the context of variable and often discordant PMASRs, our study suggests that higher consumption of dairy products may be associated with decreased risk of gastrointestinal cancer and an uncertain or no established cancer risk associated with hormone-dependent or other hormone-independent cancers. Limited data precluded our ability to evaluate the association of dairy consumption on either all-cause mortality or cancer-specific mortality.

\section{Discussion of discrepant results}

Data suggested an inconsistent but perhaps increased risk of some hormone-dependent cancers with higher dairy consumption, but also suggested some conflicting associations for breast and prostate cancer. Exogenous oestrogens from milk products consumed today ${ }^{667}$ may explain the potential increased risk in hormone-dependent cancers. The evidence pertaining to hormone-dependent cancers reported in this overview was synthesised mostly by meta-analyses performed on diverse study types (including case reports, uncontrolled cohort studies and controlled cohort studies, either retrospective or prospective) of generally low to moderate methodological quality and thus could explain the conflicting associations reported. In contrast to the hormone-dependent cancers, data suggested an inconsistent but perhaps decreased risk of gastrointestinal cancers with higher dairy consumption. In many parts of the world, dairy products consumed today are fortified with vitamin $\mathrm{D}^{68}$ and also are a rich source of calcium, conjugated linoleic acid and sphingolipids. ${ }^{41}$ Studies have suggested that high intake of dietary calcium and vitamin $\mathrm{D}$ binds to free fatty acids and secondary bile acids in the digestive tract, reducing their toxic effects on gut epithelial cells and inhibiting proliferation of intestinal mucosa and epithelial cells. ${ }^{40}$ Thus the presence of these protective factors in dairy products may contribute to the decreased risk of gastrointestinal cancers associated with higher dairy consumption. Evidence report ${ }^{6970}$ based on systematic literature review by the World Cancer Research Fund (WCRF)/American Institute for Cancer Research (AICR) reported that higher consumption of dairy products might increase risk of prostate cancer and decrease the risk of premenopausal breast cancer (limited evidence), whereas dairy products might decrease the risk of colorectal cancer (strong but probable evidence). ${ }^{69} 70$ These findings are in agreement with the conclusions of our overview of reviews for the above three cancers. In addition, in our study we have also provided evidence for the association between dairy intake and other types of cancers not addressed by the WCRF/AICR report.

Comparisons between 'no dairy consumption' and 'high/low dairy consumption' (compared with low dairy vs high dairy) may have more clearly defined the association between dairy intake and risks of hormone-dependent and gastrointestinal cancers as it is possible that the levels of reproductive hormones or protective factors in the dairy products may not have been sufficiently different between study populations consuming low dairy versus high dairy to see a significant impact of dairy on risk of cancer. 
Table 3 Nature of association between dairy consumption and risk of cancer: evidence from systematic reviews and metaanalysis

\section{Type of cancer}

\begin{tabular}{|c|c|c|c|c|c|c|c|c|c|c|c|c|c|c|}
\hline Type of dairy & CR & PR & ov & BR & GS & EP & Lung & NHL & EM & BL & PN & TD & $\begin{array}{l}\text { Renal } \\
\text { cell }\end{array}$ & MM \\
\hline \multirow[t]{4}{*}{ All-dairy products } & - & + & $\varnothing$ & - & $\varnothing$ & $\varnothing$ & $\varnothing$ & & & & & & & \\
\hline & - & & & & $\varnothing$ & & & & & & & & & \\
\hline & $\varnothing$ & & & & & & & & & & & & & \\
\hline & - & & & & & & & & & & & & & \\
\hline
\end{tabular}

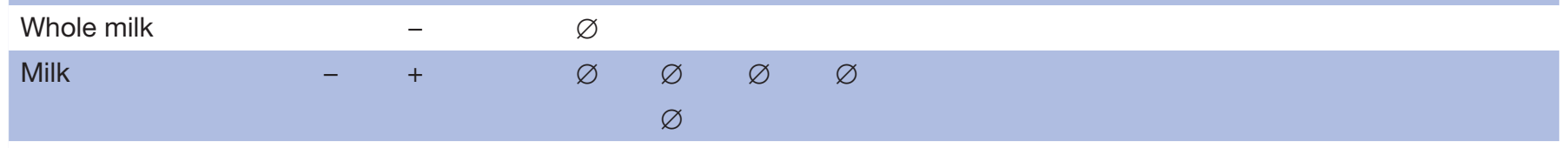

Fermented milk $\quad \varnothing$

$\varnothing$

Non-fermented milk _-

Low-fat milk

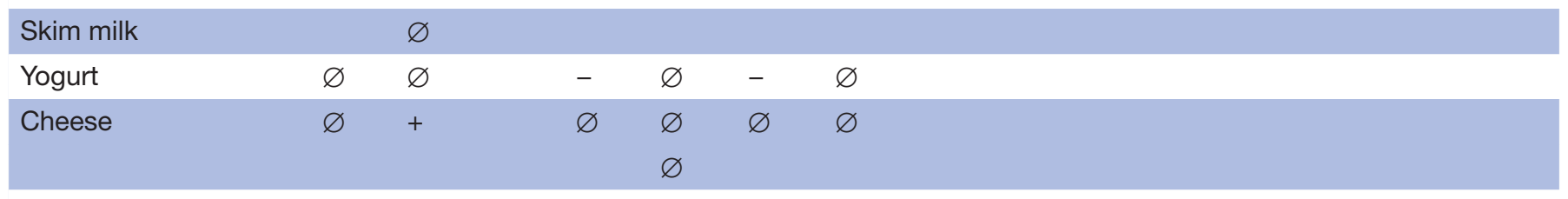

\section{Cottage cheese}

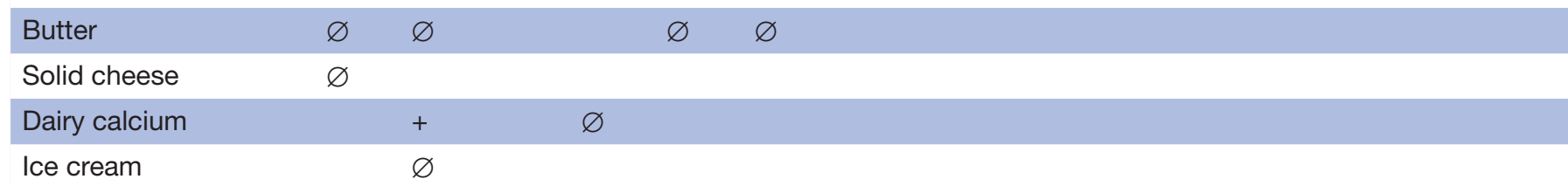

\begin{tabular}{lll} 
Fermented dairy & $\varnothing$ & \\
Low-fat dairy & $\varnothing$ & - \\
High-fat dairy & $\varnothing$ & $\varnothing$ \\
\hline
\end{tabular}

$\varnothing$, no association; -, decreased risk of cancer $(p<0.05)$; +, increased risk of cancer $(p<0.05)$; BL, bladder; BR, breast; CR, colorectal cancer; EM, endometrial; EP, oesophageal; GS, gastric; MM, multiple myeloma; NHL, non-Hodgkin's lymphoma; OV, ovary; PN, pancreas; PR, prostate; TD, thyroid.

\section{Issues of representativeness}

In this overview, among the 11 included systematic review and meta-analyses, only one ${ }^{20}$ provided evidence exclusively from prospective study designs. It is important for systematic reviews and meta-analysis to derive evidence from prospective study designs such as RCTs or prospective controlled cohort studies in order to make any inference on causal association. Thus, high-quality evidence from systematic reviews of prospective study designs at low risk of bias is needed to assess the risk of cancers associated with high dairy consumption.

\section{Issues of mortality}

The evidence of no clear association between dairy product consumption and all-cause mortality comes from one PMASR. ${ }^{64}$ Similarly, evidence for dairy consumption and cancer-specific mortality comes from only three PMASRs and requires further study. In one PMASR ${ }^{19}$ reporting significant reduction in lung cancer-specific mortality, evidence was only from three primary studies $(\mathrm{n}=42011$ participants $)$ reflecting a paucity of available data. Further primary studies and the completion of comprehensive high-quality systematic reviews are needed to explore the association between dairy consumption and risk of all-cause or cancer-specific mortality.

\section{Strengths and limitations}

This overview of reviews is the first to synthesise current evidence from PMASRs that evaluated associations between dairy consumption and risk of cancer and/or mortality. Our systematic review methods were comprehensive and our scientific protocol was established a priori and registered in PROSPERO. ${ }^{24}$ We included reviews of any date and type to provide an expansive summary of evidence on the topic. Our overview also has limitations. We only included English-language PMASRs. This may contribute to language bias and thus publication bias. The majority (12/14) of the excluded non-English 
citations did not appear to be PMASRs. As with any non-primary research, the results of this overview need to be interpreted with caution due to issues of heterogeneity. It is also important to recognise the overlap of the primary studies included in the PMASRs, the marked heterogeneity among included PMASRs with respect to included effect estimates and quality, and the predominantly retrospective nature of primary studies included in the PMASRs.

\section{CONCLUSIONS}

The association between dairy consumption and cancer risk has been explored with a variety of study designs in PMASRs of low to moderate quality. Limitations in the currently published PMASRs reduce the validity of previously published associations and mitigate assessment of causal association. To fully characterise valid associations between dairy consumption and risk of and/or cancer-related mortality, rigorously conducted systematic reviews and meta-analysis including only high-quality prospective study designs are required.

\author{
Author affiliations \\ ${ }^{1}$ The George \& Fay Yee Center for Healthcare Innovation, University of Manitoba, \\ Winnipeg, Manitoba, Canada \\ ${ }^{2}$ Department of Community Health Sciences, University of Manitoba, Winnipeg, \\ Manitoba, Canada \\ ${ }^{3}$ Max Rady College of Medicine, Rady Faculty of Health Sciences, University of \\ Manitoba, Winnipeg, Manitoba, Canada \\ ${ }^{4}$ Neil John Maclean Health Sciences Library, University of Manitoba, Winnipeg, \\ Manitoba, Canada \\ ${ }^{5}$ Department of Internal Medicine, University of Manitoba, Winnipeg, Manitoba, \\ Canada \\ ${ }^{6}$ Department of Medical Oncology and Hematology, Cancer Care Manitoba, \\ Winnipeg, Manitoba, Canada \\ ${ }^{7}$ Research Institute in Oncology and Hematology, University of Manitoba and \\ CancerCare Manitoba, Winnipeg, Manitoba, Canada
}

Acknowledgements The authors thank Christine Nielson for help with running search strategies in various databases to identify relevant studies and Amrinder Singh Mann for help with screening.

Contributors MMJ and RZ were involved in coordination of all aspects of this study, including protocol development and manuscript preparation. TG was involved in the development of search strategies for various databases. LG, LC, FF and $\mathrm{JL}$ were involved in the study selection process, data extraction and quality assessment. DD, PC and MP are experts in cancer research and provided content expertise during the design and conduct of the study. AMA-S, RZ and MMJ are experts in systematic review methodology and provided guidance on appropriate methodology for conducting this overview of reviews. All authors contributed to the design of the study and critically reviewed the manuscript.

Funding The authors have not declared a specific grant for this research from any funding agency in the public, commercial or not-for-profit sectors.

Competing interests None declared.

Patient consent for publication Not required.

Provenance and peer review Not commissioned; externally peer reviewed.

Data sharing statement Data can be obtained from the corresponding author on request.

Open access This is an open access article distributed in accordance with the Creative Commons Attribution Non Commercial (CC BY-NC 4.0) license, which permits others to distribute, remix, adapt, build upon this work non-commercially, and license their derivative works on different terms, provided the original work is properly cited, appropriate credit is given, any changes made indicated, and the use is non-commercial. See: http://creativecommons.org/licenses/by-nc/4.0/.

\section{REFERENCES}

1. Siegel RL, Miller KD, Jemal A. Cancer statistics, 2017. CA Cancer J Clin 2017;67:7-30.

2. Ferlay J, Soerjomataram I, Dikshit R, et al. Cancer incidence and mortality worldwide: sources, methods and major patterns in GLOBOCAN 2012. Int J Cancer 2015;136:E359-86.

3. American Cancer Society. Global cancer facts and figures. 3rd edn. https://www.cancer.org/research/cancer-facts-statistics/global.html.

4. Canadian Cancer Society's Advisory Committee on Cancer Statistics. Canadian cancer statistics 2017. Toronto, ON: Canadian Cancer Society, 2017. Available at. cancer.ca/Canadian-CancerStatistics-2017-EN.pdf. (accessed Jul 2017).

5. Anand P, Kunnumakkara AB, Kunnumakara AB, et al. Cancer is a preventable disease that requires major lifestyle changes. Pharm Res 2008;25:2097-116.

6. Baena Ruiz R, Salinas Hernández P. Diet and cancer: risk factors and epidemiological evidence. Maturitas 2014;77:202-8.

7. Pereira PC. Milk nutritional composition and its role in human health. Nutrition 2014;30:619-27.

8. U.S. Department of Health and Human Services and U.S, Department of Agriculture. 2015-2020 Dietary Guidelines for Americans. 8th Edition. 2015. Available at http://health.gov/ dietaryguidelines/2015/guidelines/

9. Ludwig DS, Willett WC. Three daily servings of reduced-fat milk: an evidence-based recommendation? JAMA Pediatr 2013;167:788-9.

10. Hamilton A. Got milked? How the milk kick is making you sick. 2015 https://www.chapters.indigo.ca/en-ca/books/product/ 9781443421270-item.html?ref=item_page:richrel:rr-carousel:json1

11. Hyman M. Got proof? Lack of evidence for milk's benefits. http:// drhyman.com/blog/2013/07/05/got-proof-lack-of-evidence-for-milksbenefits/

12. Pufulete M. Intake of dairy products and risk of colorectal neoplasia. Nutr Res Rev 2008;21:56-67.

13. Qin LQ, Wang PY, Kaneko T, et al. Estrogen: one of the risk factors in milk for prostate cancer. Med Hypotheses 2004;62:133-42.

14. Maruyama K, Oshima T, Ohyama K. Exposure to exogenous estrogen through intake of commercial milk produced from pregnant cows. Pediatr Int 2010;52:33-8.

15. Renehan AG, Zwahlen M, Minder C, et al. Insulin-like growth factor (IGF)-I, IGF binding protein-3, and cancer risk: systematic review and meta-regression analysis. Lancet 2004;363:1346-53.

16. Thorning TK, Raben A, Tholstrup T, et al. Milk and dairy products: good or bad for human health? An assessment of the totality of scientific evidence. Food Nutr Res 2016;60:32527.

17. Shin MH, Holmes MD, Hankinson SE, et al. Intake of dairy products, calcium, and vitamin $\mathrm{d}$ and risk of breast cancer. J Natl Cancer Inst 2002;94:1301-10.

18. Farvid MS, Malekshah AF, Pourshams A, et al. Dairy food intake and all-cause, cardiovascular disease, and cancer mortality. Am J Epidemiol 2017; 185:697-711.

19. Yang $Y$, Wang X, Yao Q, et al. Dairy product, calcium intake and lung cancer risk: a systematic review with meta-analysis. Sci Rep 2016;6:20624.

20. Aune D, Navarro Rosenblatt DA, Chan DS, et al. Dairy products, calcium, and prostate cancer risk: a systematic review and metaanalysis of cohort studies. Am J Clin Nutr 2015;101:87-117.

21. Gao X, LaValley MP, Tucker KL. Prospective studies of dairy product and calcium intakes and prostate cancer risk: a meta-analysis. JNCl: Journal of the National Cancer Institute 2005;97:1768-77.

22. Wang J, Li X, Zhang D. Dairy Product Consumption and Risk of NonHodgkin Lymphoma: A Meta-Analysis. Nutrients 2016;8:120.

23. Genkinger JM, Wang M, Li R, et al. Dairy products and pancreatic cancer risk: a pooled analysis of 14 cohort studies. Ann Oncol 2014;25:1106-15.

24. Jeyaraman MM, Abou-Setta AM, Grant L, et al. Dairy product consumption and risk of cancer - an overview of reviews. International Prospective Register of Systematic Reviews (PROSPERO). 2017 https://wwwcrdyorkacuk/prospero/display_ recordphp?RecordID=78463.

25. Liberati A, Altman DG, Tetzlaff J, et al. The PRISMA statement for reporting systematic reviews and meta-analyses of studies that evaluate health care interventions: explanation and elaboration. PLOS Med 2009;6:e1000100.

26. Shea BJ, Hamel C, Wells GA, et al. AMSTAR is a reliable and valid measurement tool to assess the methodological quality of systematic reviews. J Clin Epidemiol 2009;62:1013-20. 
27. Aune D, Lau R, Chan DS, et al. Dairy products and colorectal cancer risk: a systematic review and meta-analysis of cohort studies. Ann Oncol 2012;23:37-45.

28. Hidayat K, Chen GC, Zhang R, et al. Calcium intake and breast cancer risk: meta-analysis of prospective cohort studies. Br J Nutr 2016;116:158-66.

29. Hou R, Wu QJ, Gong TT, et al. Dietary fat and fatty acid intake and epithelial ovarian cancer risk: evidence from epidemiological studies. Oncotarget 2015;6:43099-119.

30. Li BL, Jiang GX, Xue Q, et al. Dairy consumption and risk of esophageal squamous cell carcinoma: a meta-analysis of observational studies. Asia Pac J Clin Oncol 2016;12.

31. Ralston RA, Truby H, Palermo CE, et al. Colorectal cancer and nonfermented milk, solid cheese, and fermented milk consumption: a systematic review and meta-analysis of prospective studies. Crit Rev Food Sci Nutr 2014;54:1167-79.

32. Sun Y, Lin LJ, Sang LX, et al. Dairy product consumption and gastric cancer risk: a meta-analysis. World J Gastroenterol 2014;20:15879-98.

33. Szilagyi A, Nathwani $U$, Vinokuroff $C$, et al. The effect of lactose maldigestion on the relationship between dairy food intake and colorectal cancer: a systematic review. Nutr Cancer 2006;55:141-50.

34. Tian SB, Yu JC, Kang WM, et al. Association between dairy intake and gastric cancer: a meta-analysis of observational studies. PLoS One 2014;9:e101728.

35. Zang J, Shen M, Du S, et al. The association between dairy intake and breast cancer in western and asian populations: a systematic review and meta-analysis. J Breast Cancer 2015;18:313-22.

36. Li Q, Cui L, Tian Y, et al. Protective effect of dietary calcium intake on esophageal cancer risk: a meta-analysis of observational studies. Nutrients 2017:9:18.

37. Guo Y, Shan Z, Ren H, et al. Dairy consumption and gastric cancer risk: a meta-analysis of epidemiological studies. Nutr Cancer 2015;67:555-68.

38. N-y X, J-h Z, X-b Z, et al. Association between dietary factors and stomach cancer among Chinese people: A meta analysis. Bio Medical Information Engineering 2009:179.

39. Skinner HG, Michaud DS, Giovannucci E, et al. Vitamin D intake and the risk for pancreatic cancer in two cohort studies. Cancer Epidemiol Biomarkers Prev 2006;15:1688-95.

40. Huncharek M, Muscat J, Kupelnick B. Colorectal cancer risk and dietary intake of calcium, vitamin $\mathrm{D}$, and dairy products: a metaanalysis of 26,335 cases from 60 observational studies. Nutr Cancer 2009;61:47-69.

41. Norat T, Riboli E. Dairy products and colorectal cancer. A review of possible mechanisms and epidemiological evidence. Eur J Clin Nutr 2003;57:1-17.

42. Rosato V, Guercio V, Bosetti C, et al. Mediterranean diet and colorectal cancer risk: a pooled analysis of three Italian case-control studies. Br J Cancer 2016;115:862-5.

43. Cho E, Smith-Warner SA, Spiegelman D, et al. Dairy foods, calcium, and colorectal cancer: a pooled analysis of 10 cohort studies. $J$ Natl Cancer Inst 2004:96:1015-22.

44. Huncharek M, Muscat J, Kupelnick B. Dairy products, dietary calcium and vitamin $D$ intake as risk factors for prostate cancer: a meta-analysis of 26,769 cases from 45 observational studies. Nutr Cancer 2008;60:421-41.

45. Qin LQ, Hy X, Wang PY, et al. Milk consumption is a risk factor for prostate cancer in Western countries: evidence from cohort studies. Asia Pac J Clin Nutr 2007;16:467-76.

46. Gao X, LaValley MP, Tucker KL. Prospective studies of dairy product and calcium intakes and prostate cancer risk: a meta-analysis. $J$ Natl Cancer Inst 2005;97:1768-77.

47. Epstein MM, Smith-Warner SA. Risk of prostate cancer with intake of dietary and total calcium: A pooled analysis of 14 prospective cohort studies. Cancer Prevention Research Conference: AACR International Conference on Frontiers in Cancer Prevention Research 2011:4.
48. Qin LQ, Xu JY, Wang PY, et al. Milk consumption is a risk factor for prostate cancer: meta-analysis of case-control studies. Nutr Cancer 2004;48:22-7.

49. Lane JA, Oliver SE, Appleby PN, et al. Prostate cancer risk related to foods, food groups, macronutrients and micronutrients derived from the UK Dietary Cohort Consortium food diaries. Eur J Clin Nutr 2017:71:274-83.

50. Larsson SC, Orsini N, Wolk A. Milk, milk products and lactose intake and ovarian cancer risk: a meta-analysis of epidemiological studies. Int J Cancer 2006;118:431-41.

51. Qin LQ, Xu JY, Wang PY, et al. Milk/dairy products consumption, galactose metabolism and ovarian cancer: meta-analysis of epidemiological studies. Eur J Cancer Prev 2005;14:13-19.

52. Genkinger JM, Hunter DJ, Spiegelman D, et al. Dairy products and ovarian cancer: a pooled analysis of 12 cohort studies. Cancer Epidemiol Biomarkers Prev 2006;15:364-72.

53. Liu J, Tang W, Sang L, et al. Milk, yogurt, and lactose intake and ovarian cancer risk: a meta-analysis. Nutr Cancer 2015;67:68-72.

54. Filomeno M, Bosetti C, Bidoli E, et al. Mediterranean diet and risk of endometrial cancer: a pooled analysis of three Italian case-control studies. Br J Cancer 2015;112:1816-21.

55. Missmer SA, Smith-Warner SA, Spiegelman D, et al. Meat and dairy food consumption and breast cancer: a pooled analysis of cohort studies. Int J Epidemiol 2002;31:78-85.

56. Boyd NF, Martin LJ, Noffel M, et al. A meta-analysis of studies of dietary fat and breast cancer risk. Br J Cancer 1993;68:627-36.

57. Li F, An SL, Zhou Y, et al. Milk and dairy consumption and risk of bladder cancer: a meta-analysis. Urology 2011;78:1298-305.

58. Mao QQ, Dai $Y$, Lin YW, et al. Milk consumption and bladder cancer risk: a meta-analysis of published epidemiological studies. Nutr Cancer 2011;63:1263-71.

59. Lee JE, Hunter DJ, Spiegelman D, et al. Intakes of coffee, tea, milk, soda and juice and renal cell cancer in a pooled analysis of 13 prospective studies. Int J Cancer 2007;121:2246-53.

60. Yu Y, Li H, Xu K, et al. Dairy consumption and lung cancer risk: a meta-analysis of prospective cohort studies. Onco Targets Ther 2016;9:111-6.

61. Caini S, Masala G, Gnagnarella P, et al. Food of animal origin and risk of non-Hodgkin lymphoma and multiple myeloma: a review of the literature and meta-analysis. Crit Rev Oncol Hematol 2016;100:16-24.

62. Wang J, Li X, Zhang D. Dairy product consumption and risk of NonHodgkin Lymphoma: a meta-analysis. Nutrients 2016;8:120.

63. Franceschi S, Levi F, Negri E, et al. Diet and thyroid cancer: a pooled analysis of four European case-control studies. Int $\mathrm{J}$ Cancer 1991;48:395-8

64. O'Sullivan TA, Hafekost K, Mitrou F, et al. Food sources of saturated fat and the association with mortality: a meta-analysis. Am J Public Health 2013;103:e31-42.

65. Lu W, Chen H, Niu Y, et al. Dairy products intake and cancer mortality risk: a meta-analysis of 11 population-based cohort studies. Nutr $\mathrm{J}$ 2016;15:91

66. Ganmaa D, Wang PY, Qin LQ, et al. Is milk responsible for male reproductive disorders? Med Hypotheses 2001;57:510-4.

67. Ganmaa D, Sato A. The possible role of female sex hormones in milk from pregnant cows in the development of breast, ovarian and corpus uteri cancers. Med Hypotheses 2005;65:1028-37.

68. Calvo MS, Whiting SJ, Barton CN. Vitamin D fortification in the United States and Canada: current status and data needs. Am J Clin Nutr 2004;80:1710S-6.

69. World Cancer Research Fund/American Institute for Cancer Research. Diet, nutrition, physical activity and cancer: a global perspective. Continuous update project expert report. 2018. Available at dietandcancerreport.org (Accessed 09 Nov 2018).

70. World Cancer Research Fund/American Institute for Cancer Research. Continuous update project expert report 2018. Meat, fish and dairy products and the risk of cancer. Available at dietandcancerreport.org (Accessed 09 Nov 2018). 